\title{
Effects of Stimulus Onset Asynchrony on Parafoveal Presentation of Homographs
}

\author{
Jeremy William Grabbe, Michelle Mann-Saumier, \\ Kaeli Entrup and Autumn M. Gooden-Malone
}

Department of Psychology, State University of New York, SUNY Plattsburgh, 101 Broad Street, Plattsburgh, 12901, New York

Received 2012-12-22, Revised 2012-12-23; Accepted 2012-12-23

\begin{abstract}
Recent research in word recognition has found a benefit for right parafoveal presentation. This asymmetrical finding has lead to a need to reexamine previous research in foveal-parafoveal word processing. This study reexamined previous research on homographs and the parafovea, previously no parafoveal effect/benefit was found. The authors in the current study attempted to replicate Inhoff's original work by compensating for possible methodological limitations in Inhoff's study. The results showed that Stimulus Onset Asynchrony (SOA) was a factor in the original Inhoff study. The results of the current study show that when controlling for SOA a parafoveal benefit did exist.
\end{abstract}

Keywords: Parafovea Preview, Homographs

\section{INTRODUCTION}

\subsection{Effects of Stimulus Onset Asynchrony on Parafoveal Presentation of Homographs}

Grabbe and Allen (2012) found that older adults did not perform significantly different for a lexical decision task when a lexical stimulus was presented in the right parafoveal area of the visual field. This finding of anyone having parafoveal lexical processing was unusual, even more so since the same effect was found on older as well as younger adults. However, other studies (Paterson et al., 2012; Calvo and Lang, 2005) are also starting to show a benefit for parafoveal processing. This effect is usually found the right parafovea for native English speakers because they read left to right.

It has now become necessary to revisit previous studies of parafoveal processing which did not show parafoveal benefits. Given these new findings, previous studys may not have found comparable results due to limitations of methodology. Inhoff (1982) conducted a study that did not find semantic priming in the parafovea. A possible reason that Inhoff did not find parafoveal effects is that the methodology had homographs and target words appear in close temporal proximity. Neo and Chua (2006) showed that if there is a sudden onset of a distracter it will affect performance. The close temporal presentation of target and homographs may have lead to a quasi-distracter effect in Ihnoff's study. Replicating Inhoff's study and finding parafoveal semantic priming would be more congruent to the results of Grabbe and Allen (2012).

A plausible reason exists that could explain why Inhoff (1982) did not find semantic priming and why Grabbe and Allen (2012) found parafoveal processing. That explanation is lies in the methodology employed by Inhoff. Inhoff's methodology consisted of presenting a homograph (a word that can have multiple meanings) at the same time as a word that corresponds to the semantic meaning of one of the meanings of the foveally presented homograph. The participant was then asked to define the homograph that was presented in the fovea. If the participant's definition of the word was similar to the 101 Broad Street, Plattsburgh, 12901, New York 
meaning of the parafoveal word then that was taken as evidence of parafoveal processing.

A key point to be made about this is that the presentation of the words occurred simultaneously. In a dual task setting there is a PRP effect which is a refractory period in processing between two tasks when the first task takes up a central bottle neck and the second task (Allen et al., 1998). During this refractory period task 2 cannot be processed. When the SOA between tasks are increased the PRP effect is lessened. If Inhoff had an SOA of zero then it is probable that the lack of semantic priming was not due to an inability to process information parafoveally, but a product of the PRP effect preventing processing of the parafoveal word.

This study shall replicate the works of Inhoff (1982) and seek to demonstrate parafoveal semantic priming by manipulating the SOA between the Foveal and parafoveal word. Inhoff's study was replicated with SOA being manipulated to demonstrate that Inhoff's findings were more likely the result of PRP effects and not a lack of processing. The methodology of Inhoff is comparable to a dual-task with PRP effects.

\section{MATERIALS AND METHODS}

\subsection{Participants}

Participants were eight undergraduates from the State University of New York, Plattsburgh. Five participants were female. The mean age for participants was 22.3 years of age and all were right handed. Participants receive course credit for participation.

\subsection{Stimuli}

One hundred and forty-four homographs were selected for the homograph condition and an equal number of unambiguous words were selected for the nonhomograph condition. Strings of letters were presented beginning at 1 degree (foveal) and 3 degrees (parafoveal) of visual angle, both of which were presented equally to the left and right. In order to ensure equal retinal eccentricities, right presentations involved the presentation of the first letter at 1 degree (foveal) or 3 degrees (parafoveal), whereas left presentation involved the last letter of a stimulus appearing at 1 degree or 3 degrees.

\subsection{Procedure}

On a computer screen two words were presented in quick succession. The stimulus onset asynchrony between the words was 50, 200, or 500 milliseconds. They will appear on different sides of the screen (Left Parafovea, Left Fovea, Right Fovea and Right Parafovea). Immediately after the words were presented two sentences will be presented on the screen. Participants will select the sentence that connects the meaning between the words. For example the words "exercise" and "nature" will be presented on the screen. Afterwards then two phases will appear on the screen such as "To take a walk outside." and "To watch." The phase that would connect the two words would be "To take a walk outside."

\section{RESULTS}

\subsection{Reaction Time}

Data was analyzed in a $2 \times 3 \times 4$ (homograph Vs nonhomograph $\times \mathrm{SOA} \times$ location) repeated measures analysis of variance. No responses below $300 \mathrm{~ms}$ were included in the analysis

There was a main effect of location, $F(3,21)=$ $4.157, \mathrm{p}<0.05$ with quicker reaction times in the fovea and right parafovea. There was a significant three-way interaction between homograph $\times \mathrm{SOA} \times$ location, $\mathrm{F}(6,42)=2.386, \mathrm{p}<0.05$. This was a result of a decrease in reaction time for the right fovea and right parafovea for homographs as SOA increased. By contrast, nonhomographs retained comparable levels of performance regardless of SOA or location (with the exception of lower reaction times in the fovea which were not dramatically affected by SOA; Fig. 1).

\subsection{Accuracy}

Accuracy data showed a main effect for SOA, F(2, $14)=19.03, p<0.05$. Accuracy increased as SOA increased. There was a significant main effect for location, $\mathrm{F}(3,21)=8.03, \mathrm{p}<0.05$ (Left Parafovea $=$ $66.5 \%$, Left Fovea $=80.4 \%$, Right Fovea $=82.0 \%$, Right Parafovea $=71.4 \%$ ). Homograph and location showed a significant interaction, $\mathrm{F}(2,14)=6.63, \mathrm{p}<0.05$.

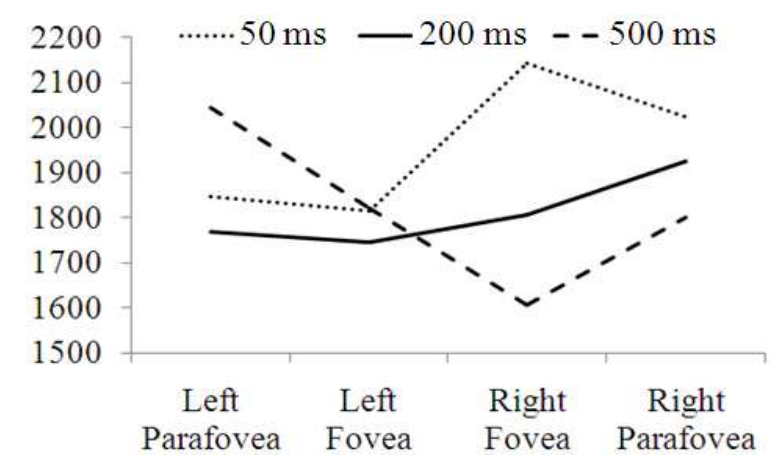

Fig. 1. Mean Percent Accuracy as a Function of SOA and Location (Left Parafoveal, Left Foveal, Right Foveal, Right Parafoveal) 


\section{DISCUSSION}

As new discoveries in the field of word recognition, reading and retinal location show a more potent role of the parafovea, it is important to reexamine past studies of fovea-parafovea word recognition. This study reexamined Inhoff (1982) study of homographs and the parafovea. This new study examined the role of SOA as a factor in perception. It was found that SOA can either lead to parafoveal presentation to serve as a distracter (at short SOA; Neo and Chua, 2006) or facilitate performance (at long SOA; Grabbe and Allen, 2012).

\section{CONCLUSION}

This study contributes to a growing number of studies showing benefits of parafoveal presentation (Grabbe and Allen, 2012; Paterson et al., 2012; Calvo and Lang, 2005) by actually showing parafoveal semantic preprocessing. This demonstrates that earlier studies that did not find parafoveal benefit may have been the result of methodological limitations such as SOA. It can be suggested that revisiting earlier studies' methodologies may have greater benefit for contemporary researchers in the field of visual word recognition.

\section{REFERENCES}

Allen, P.A., A.F. Smith, H. Vires-Collins and S. Sperry, 1998. The psychological refractory period: Evidence for age differences in attentional time-sharing. Psychol. Aging, 13: 218-229. DOI: 10.1037/08827974.13.2.218
Calvo, M.G. and P.J. Lang, 2005. Parafoveal semantic processing of emotional visual scenes. J. Exp. Psychol. Hum. Perception Perform., 31: 502-519. DOI: $10.1037 / 0096-1523.31 .3 .502$

Grabbe, J.W. and P.A. Allen, 2012. Cross-task compatibility and age-related dual-task performance. Exp. Aging Res.: Int. J. Devoted Sci. Study Aging Process, 38: 469-487. DOI: 10.1080/0361073X.2012.726154

Inhoff, A.W., 1982. Parafoveal word perception: A further case against semantic preprocessing. J. Exp. Psychol. Hum. Percept. Perform., 8: 137-145. DOI: 10.1037/0096-1523.8.1.137

Neo, C. and F.K. Chua, 2006. Capturing focused attention. Percept. Psychophy., 68: 1286-1295. DOI: 10.3758/BF03193728

Paterson, K.B., V.A. McGowan and T.R. Jordan, 2012. Filtered text reveals adult age differences in reading: Evidence from eye movements. Psychol. Aging. PMID: 23066801 